\title{
METHODS OF CONSTRUCTING HYPERFIELDS
}

\section{CH. G. MASSOUROS}

54 Klious St. Cholargos

Ahtens, Greece

TK 155-61

(Received February 1, 1983 and in revised form July 21, 1983)

ABSTRACT. In this paper we introduce a class of hyperfields which contains non quotient hyperfields. Thus we give a negative answer to the question of whether every hyperfield is isomorphic to a quotient $\frac{K}{G}$ of a field $K$ by some subgroup $G$ of its multiplicative group.

KEY WORDS AND PHRASES. Hypercomposition, hyperfield, quotient hyperfield, hyperring. 1980 AMS SUBJECT CLASSIFICATION CODE. $12 \mathrm{Kg}$.

\section{INTRODUCTION}

A hyperfield is a triplet $(\mathrm{H},+, \cdot)$ where $\mathrm{H}$ is a non-empty set, + is a hypercomposition (i.e. a mapping whose domain is $\mathrm{H} \times \mathrm{H}$ and whose range is the power set of $H$ ), and - an internal composition of $H$ (i.e. for every $x, y \in H, x \cdot y \in H$ ). These two operations satisfy the following axioms:

I. Multiplicative axiom

$\mathrm{H}$ is an almost-group with regard to the multiplication. An almost-group is a semigroup $S$, which is the union $G U\{0\}$, where $G$ is a group and 0 a bilaterally absorbing element of $\mathrm{S}$. By 1 we shall denote its neutral element and by 0 its absorbing element.

II. Additive axioms

(i) $\mathrm{x}+\mathrm{y}=\mathrm{y}+\mathrm{x}$

(ii) $(\mathrm{x}+\mathrm{y})+\mathrm{z}=\mathrm{x}+(\mathrm{y}+\mathrm{z})$

(iii) For every $x \in H$, there exists one and only one $x^{\prime} \epsilon H$ such that $0 \epsilon x^{\prime}+x^{\prime}$. $x^{\prime}$ is written $-x$ and called the opposite of $x$; moreover we shall write

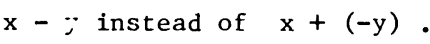

(iv) $z \in \mathrm{x}+\mathrm{y}$ implies that $\mathrm{y} \in \mathrm{z}-\mathrm{x}$

III. Distributive axiom $z \cdot(x+y)=z \cdot x+z \cdot y,(x+y) \cdot z=x \cdot z+y \cdot z$

We remark that:

(a) By usual convensions if "." is an internal composition of a set $E$ and $X, Y$ are 
subsets of $E, X \cdot Y$ is the set of all $x \cdot y$ such that $(x, y) \in X \times Y$. If "." is a hypercomposition in $E$ then $X \cdot Y$ signifies the union $U(x, y) \epsilon X \times Y X \cdot y$. In both cases $\mathrm{X} \cdot \mathrm{Y}$ and $\mathrm{X} \cdot \mathrm{y}$ will have the same meaning as $\{\mathrm{x}\} \cdot \mathrm{Y}$ and $\mathrm{X} \cdot\{\mathrm{y}\}$ respectively.

(b) By virtue of axioms II. (iii) and II.(iv) one has

$$
\mathrm{x}+0=\mathrm{x} \text { for each } \mathrm{x} \in \mathrm{H}
$$

Indeed; $0 \in \mathrm{x}-\mathrm{x}$ so $\mathrm{x} \in \mathrm{x}+0$. Let's suppose that some $\mathrm{y}$ different than $\mathrm{x}$ belongs to $x+0$. Then $0 \in \mathrm{x}-\mathrm{y}$ which is absurd because of II.(iii). So $\mathrm{x}+0=\mathrm{x}$.

Also, because + is a hypercomposition, the distributive axiom gives:

$(a+b)(c+d) \subseteq a c+a d+b c+b d$. (properties of hyperfields can be found in [3],

[5])

(c) If we replace the multiplicative axiom I by

$I^{\prime}$. $\mathrm{H}$ is a multiplicative semigroup having a bilaterally absorbing element 0 . we obtain a more general struccure $(\mathrm{H},+, \cdot)$ which is called hyperring. (see [4], [6])

The concept of the hyperfield has been introduced by M. Krasner in his study [1].

The hyperfield that occurs there was obtained by considering a quotient of the type

$\frac{K}{\pi_{\rho}}$ where $K$ is a valued field and $\pi_{\rho}$ an equivalence relation defined from the

field's valuation. This hyperfield was called the residual of $\mathrm{K}\left(\bmod \pi_{\rho}\right)$.

2. THE QUOTIENT HYPERFIELD.

Let $F$ be a field and $G$ a subgroup of $F^{\prime} s$ multiplicative group. Then the multiplicative classes modulo $G$ in $F$ form a partition of $F$. We shall denote by $\bar{F}=\frac{F}{G}$ the set of the classes of this partition. A multiplication can be introduced in $\bar{F}$ by defining the product $\bar{x} \cdot \bar{y}$ of two classes $\bar{x}, \bar{y}$ to be the class which results from their setwise product. It can also be proved (see [2]) that if we add $\bar{x}, \bar{y}$ as subsets of $F$, the set that results is a union of classes modulo $G$. This enables us to define the sum $\bar{x}+\bar{y}$ of $\bar{x}, \bar{y}$ to be the set of all classes $\bar{z} \epsilon \bar{F}$ which are contained in the setwise sum of $\bar{x}, \bar{y} \cdot \bar{F}$ endowed with these two operations becomes a hyperfield ([2]) called quotient hyperfield of $F$ by $G$.

If $F$ is a ring and $G$ a normal subgroup of $F^{*}(:=F-\{0\})$ (that is a subset of $R$, such that $(G, \cdot)$ is a group, and $x G=G x$ for all $x$ in $R$ ) then $\bar{F}=\frac{F}{G}$ will be a hyperring called the quotient hyperring of $F$ by $G$.

The question that Krasner sets ([2])is whether there are other hyperfields besides the quotient hyperfields. Indeed it is quite important to find out how rich the class of all hyperfields is, for if there were only quotient hyperfields then a good part of hyperfield theory could have been deduced directly from corresponding results in field theory.

At first one can notice that the residual hyperfields are always quotient hyperfields. Next we shall prove that every subhyperfield of a quotient hyperring is a quotient of a field by a multiplicative group. Indeed let $\frac{R}{G}$ be a hyperring and $\frac{F}{G}$ a subhyperfield of $\frac{R}{G}$. Then

LEMMA 1. If $u G$ is the unit of $\frac{F}{G}$, then $u G$ is a multiplicative group. 
PROOF. Let $\mathrm{x}=\mathrm{u} \gamma$ be an element of $\mathrm{uG}$. Since $\mathrm{uG}=\mathrm{Gu}$ we have: $\mathrm{xuG}=(\mathrm{u} \gamma) \mathrm{uG}=\mathrm{u}\left(\mathrm{u}_{\gamma^{\prime}}\right) \mathrm{G}=\mathrm{u}^{2} \mathrm{G} \subseteq \mathrm{uG} \cdot \mathrm{uG}=\mathrm{uG}$. So the class $\mathrm{xuG}$ is a subset of $u G$, thus $\mathrm{xuG}=\mathrm{uG}$. In the same way we can show that $\mathrm{uGx}=\mathrm{uG}$ and therefore $\mathrm{uG}$ is indeed a group.

Now if we denote by [[FG]] the subring of $R$ which is generated by the set $\mathrm{FG}$, we observe that $[[F G]]=u G[[F G]\rfloor=[[F G]] \mathrm{uG}$.

LEMMA 2. The unit of $u G$ is a]so the unit of [[FG]]

PROOF. Let $e$ be the unit of $u G$. If $s \in[[F G]]$ then there exist $s^{\prime} \epsilon$ $[[F G]]$ and $\gamma \in G^{\prime}$ such that $s=s^{\prime}\left(u_{\gamma}\right)$. Thus we have: $s e=\left(s^{\prime}(u \gamma)\right) e=s^{\prime}((u \gamma) e)=$ $=s^{\prime}(u \gamma)=s$. Similarly es $=s$ and so $e$ is the unit of $[[F G]]$.

PROPOSITION 1. Let $R$ be a ring, $G$ a normal multiplicative subgroup of $R^{*}$ and $\frac{R}{G}$ the quotient hyperring of $R$ by $G$. Then every subhyperfield of $\frac{R}{G}$ is the quotient of a subfield of $R$ by some subgroup of this subfield's multiplicative group.

PROOF. Let $\frac{F}{G}$ be a subhyperfield of $\frac{R}{G}$ and let $u G$ be its unit. We observe that $\frac{F}{G}=\frac{[[F G]]}{u G}$. Now since $\frac{[[F G]]}{u G}$ is a hyperfield, for every $t \in[[F G]]$ there exists an element $t^{\prime} \epsilon\left[[F G]\right.$ such that $t G \cdot t^{\prime} G=u G$, and therefore there exist $\gamma, \gamma^{\prime} \in G$ such that $(t \gamma)\left(t^{\prime} \gamma^{\prime}\right)=e$, where $e$ is both the unit of $G$ and the unit of $[[F G]]$. Thus $\gamma t^{\prime} \gamma^{\prime}$ is the inverse element of $t$ and therefore [[FG]] is a field.

3. NON QUOTIENT HYPERFIELDS.

We shall construct a class of hyperfields which contains hyperfields that are not isomorphic to quotient ones. For this purpose we consider a commutative multiplicative almost-group $(\mathrm{H}, \cdot)$ in which we introduce a hypercomposition + defined as follows:

and

$$
\begin{array}{ll}
x+y=\{x, y\} & \text { if } x, y \neq 0 \text { and } x \neq y \\
x+x=H \backslash\{x\} & \text { if } x \neq 0
\end{array}
$$

Then:

PROPOSITION 2. The triplet $(\mathrm{H},+, \cdot)$ is a hyperfield.

PROOF. At first one can notice that the opposite of $x$ is $x$ itself since $0 \epsilon \mathrm{x}+\mathrm{x}$. Next it is obvious that the hypercomposition is commutative and that the associativity is valid when one of $x, y, z$ is equal to 0 . Now if $x, y, z \neq 0$ and distinct, then we have:

$$
x+(y+z)=x+\{y, z\}=(x+y) U(x+z)=\{x, y\} U\{x, z\}=\{x, y, z\}
$$

A similar calculation shows $(x+y)+z=\{x, y, z\}$. Finally if two of $x, y, z$ are equal to each other; for example $x=y$, we have:

$$
\begin{aligned}
& \mathrm{x}+(\mathrm{y}+\mathrm{z})=\mathrm{x}+(\mathrm{x}+\mathrm{z})=\mathrm{x}+\{\mathrm{x}, \mathrm{z}\}=(\mathrm{x}+\mathrm{x}) \mathrm{U}(\mathrm{x}+\mathrm{z})=[\mathrm{H} \vee\{\mathrm{x}\}] \mathrm{U}\{\mathrm{x}, \mathrm{z}\}=\mathrm{H} \\
& \text { and } \quad(x+y)+z=(x+x)+z=[H \backslash\{x\}]+z \geq(z+z) U(w+z)= \\
& =[H \backslash\{z\}] U\{w, z\}=H \text {, with } w \neq z
\end{aligned}
$$

Now as far as the axiom II.iv. is concerned we have:

(i) if $y=x$ then $x+y=x+x=H \backslash\{x\}$. This means that for every $z \in H \backslash\{x\}$ we must have $x \in z+x$ which is in fact true. 
(ii) If $y \neq x, 0$ then $x+y=\{x, y\}$, thus $z=x$ or $z=y$. If $z=x$ then $x \in z+y=\{z, y\}$ and if $z=y$ then $x \in z+y=H \backslash\{y\}$. So this axiom is valid as well.

Finally it is straight forward to verify the distributive axiom.

THEOREM. The class of the hyperfields constructed as above contains elements that do not belong to the class of quotient hyperfields.

PROOF. We choose an almost group $(\mathrm{H}, \cdot)$ with card $\mathrm{H}>3$ and such that $\mathrm{x}^{2}=1$ for every $\mathrm{x} \in \mathrm{H} \backslash\{0\}$. Next we consider the hyperfield $(\mathrm{H},+, \cdot)$ which is constructed according to the above method and we suppose that this hyperfield is isomorphic to a quotient hyperfield $\left(\frac{F}{G}+\cdot\right)$ Then for $\frac{F}{G}$ the following must be valid:

(i) $\mathrm{xG} \cdot \mathrm{xG}=\mathrm{G}$ or $\mathrm{x}^{2} \mathrm{G}=\mathrm{G}$ for every $\mathrm{x} \in \mathrm{F}$

(ii) $\quad G=-G$

(iii) $G+G=\left(\frac{F}{G}\right) \backslash G$ from which $G+G=F \backslash G$

Because of (i) $G$ must contain all the squares of $F$. But if $F$ is not of characteristic 2 then every element of $F$ can be written as a difference of two squares:

$$
x=\left(\frac{x+1}{2}\right)^{2}-\left(\frac{x-1}{2}\right)^{2}=\left(\frac{x+1}{2}\right)^{2}+(-1)\left(\frac{x-1}{2}\right)^{2}
$$

So we have $G-G=G+G=F$. This contradicts (iii).

Now if the characteristic of $F$ is 2 then the sum of two squares is always a square; thus $G \subseteq G+G$, which contradicts again (iii). So $H$ cannot be isomorphic to any quotient hyperfield.

REMARKS. (a) This theorem gives something more, that is, $H$ cannot be isomorphic even to a subhyperfield of a quotient hyperring.

(b) Two other classes of hyperfields completely different can be found in [7]. The problem of those hyperfields' isomorphism to quotient hyperfields is also discussed there but no final answer is given.

\section{REFERENCES}

1. KRASNER, M. Approximation des corps values complete de caracteristique $p \neq 0$ par ceux de caracteristique 0. Colloque d' Algebre Superieure (Bruxelles, Decembre 1956), CBRM, Bruxelles 1957.

2. KRASNER, M. A class of hyperrings and hyperfields. Internat. J. Math. Math. Sci. $\underline{6}$ (1983) 307-312.

3. MITTAS, J. : Sur les hyperanneaux et les hypercorps Mathematica Balkanica, t. 3. (1973) $368-382$.

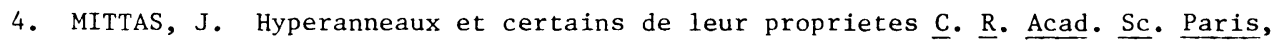
Serie A t. 269 (1969)623-626.

5. STRATIGOPOULCS, D. Hyperanneaux, hypercorps, hypermodules, hyperspaces vectoriels et leurs proprietes elementaires, $\underline{C}$. ‥ Acad. Sc. Paris, Serie A, t. 269 (1969) 489-492.

6. STRATIGOPOULOS, D. Hyperanneaux artiniens, centralisateur d'un hypermodule et theoreme de densite, $\underline{C}$. R. Acad. Sc. Paris, Serie $\underline{A}$, t. 269 (1969) 889-891.

7. STRATIGOPOULOS, D. and MASSOUROS, Ch. G. : On a class of fields Mathematica Balkanica, t. 12 . 


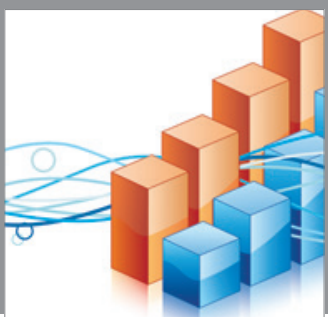

Advances in

Operations Research

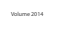

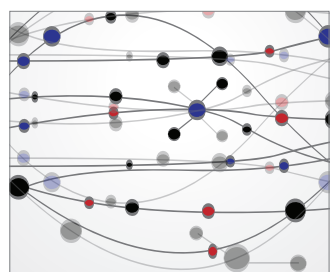

\section{The Scientific} World Journal
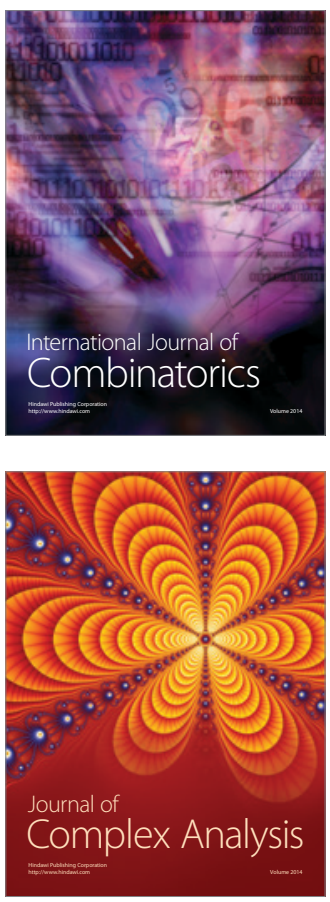

International Journal of

Mathematics and

Mathematical

Sciences
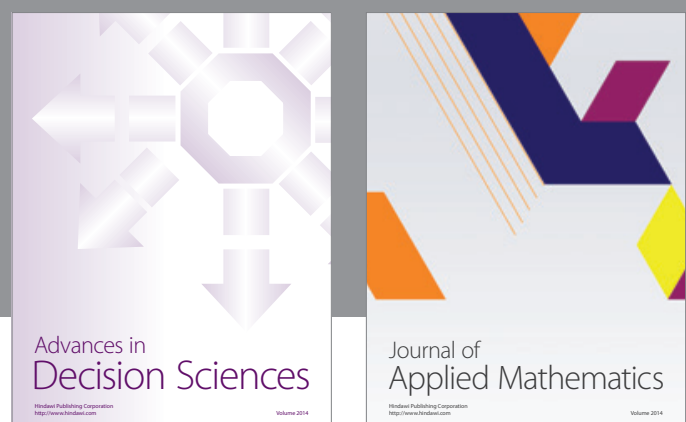

Journal of

Applied Mathematics
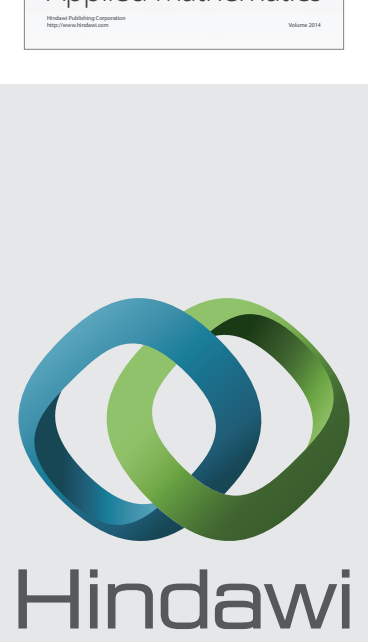

Submit your manuscripts at http://www.hindawi.com
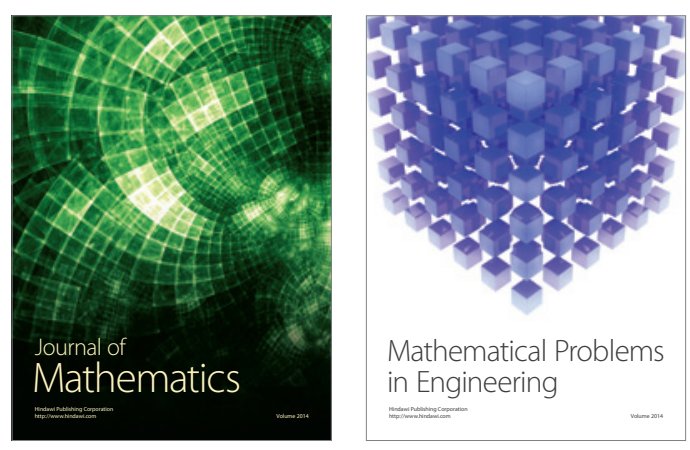

Mathematical Problems in Engineering
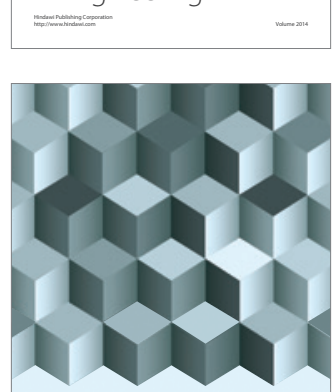

Journal of

Function Spaces
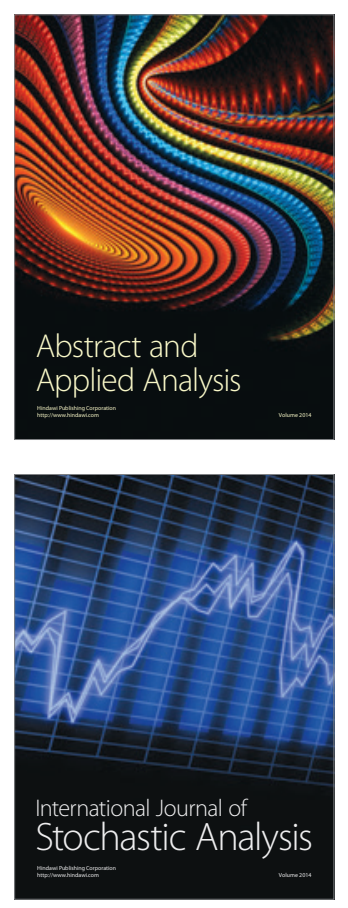

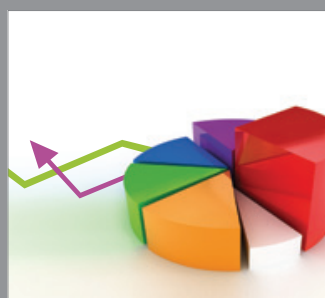

ournal of

Probability and Statistics

Promensencen
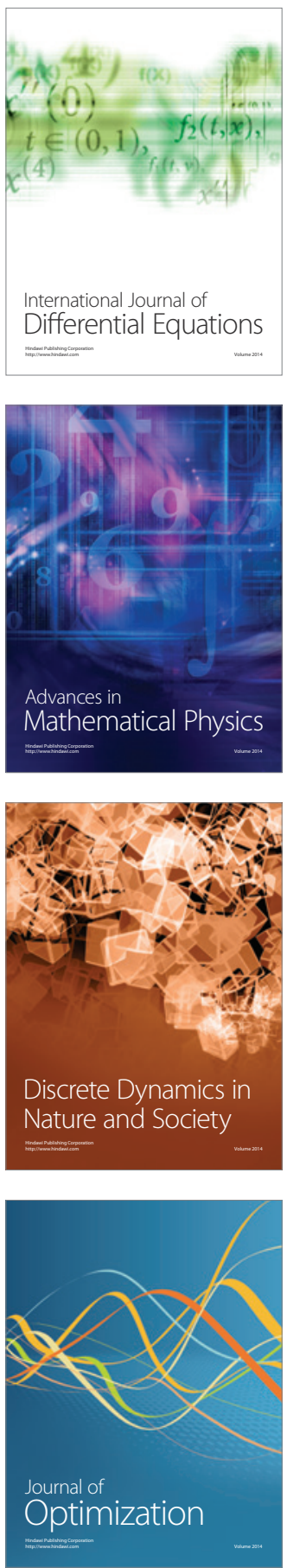